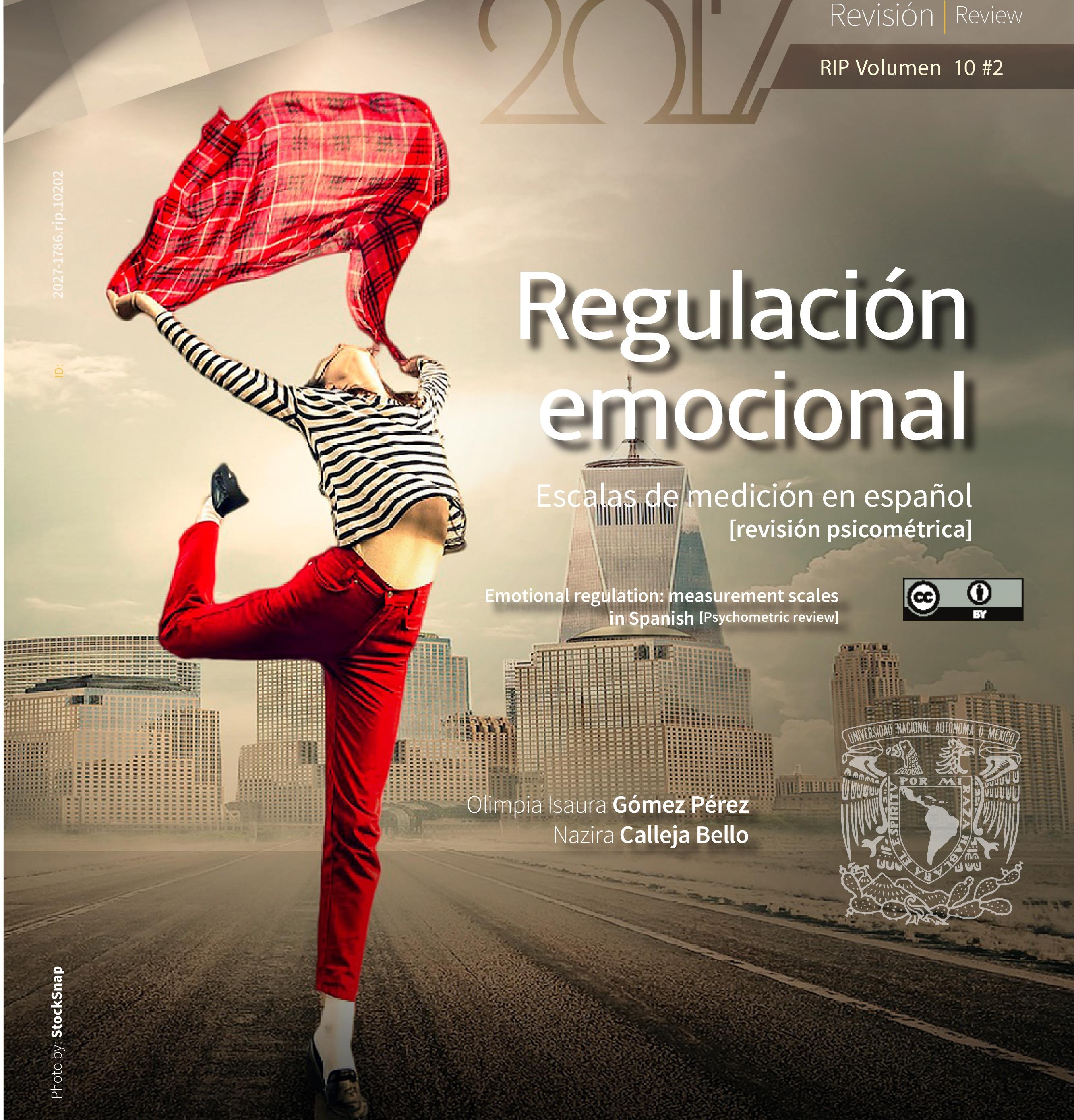




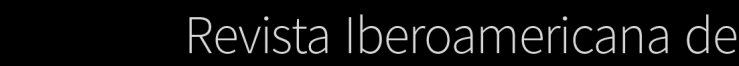 Psicología ISSN-I: 2027-1786 | e-ISSN: 2500-6517 Publicación Semestral}

ID:

\section{7-1786.rip.10202}

Title:

Subtitle: Measurement scales in Spanish [psychometric review]

Título: Regulación emocional

Subtítulo: Escalas de medición en español [revisión psicométrica]

Alt Title / Título alternativo:

[en]: $\quad$ Scales in spanish of measurement of emotional regulation: psychometric review

[es]: $\quad$ Escalas de medición en español de la regulación emocional: revisión psicométrica

Author (s) / Autor (es):

\section{Gómez Pérez, \& Calleja Bello}

Keywords / Palabras Clave:

emotional regulation; measurement; spanish speaking population; validity; reliability

[es]: $\quad$ regulación emocional; medición; población hispanohablante; validez; confiabilidad

Submited: 2017-05-29

Acepted: 2017-08-24

\section{Resumen}

Se revisó el rigor psicométrico de escalas de Regulación Emocional (RE) utilizadas en población hispanohablante. Se analizaron bases de datos electrónicas, localizando artículos de revistas especializadas de Latinoamérica y España que reportaran la construcción o adaptación de instrumentos de RE en español; posteriormente, se describió su propósito, definición de RE y principales características; finalmente, se evaluó su calidad psicométrica. De 24 escalas, siete fueron construidas en español y 17 adaptadas de 10 originales en inglés, dirigidas tanto a niños como a adolescentes y adultos, con una media de 33 reactivos y cinco dimensiones, y aplicadas

a un promedio de 320 participantes. Las principales estrategias de regulación

emocional fueron las cognitivas. La validez de constructo resultó el criterio psicométrico

más reportado. Los puntajes de calidad

fueron más altos para escalas originales en

inglés. Las escalas mejor puntuadas fueron las adaptaciones de la DERS, ERQ y CERQ y el ERQ en inglés.

Olimpia Isaura Gómez Pérez, MA

ORCID: $\quad$ 0000-0001-7264-0398

Source I Filiacion:

Universidad Nacional Autónoma de México

$\mathrm{BIO}$

Maestra en Psicología Escolar

City I Ciudad

México DF $[\mathrm{mx}]$

e-mail:

olimpiawhite@correo.unam.mx

\section{Abstract}

The psychometric rigor of Emotional Regulation (ER) scales used in the Spanishspeaking population was reviewed. Electronic databases were analyzed, locating articles from specialized journals in Latin America and Spain that reported the construction or adaptation of ER instruments in Spanish. Later, its purpose, definition of ER and main characteristics were described; finally, their psychometric quality was evaluated.

of 24 scales, seven were constructed in

Spanish and 17 adapted from 10 originals in English, aimed at children, adolescents,

and adults, with an average of 33 items and five dimensions, applied to an average

of 320 participants. The main strategies of emotional regulation were the cognitive ones. Construct validity resulted the psychometric criterion more reported. Quality scores were higher for original scales in English. The best scores were for the adaptations of the DERS, ERQ and CERQ scales, and the ERQ in English.

Nazira Calleja Bello, PhD

ORCID: $\quad$ 0000-0003-0131-8785

Source I Filiacion

Universidad Nacional Autónoma de México

BIO:

PhD en Psicología

Investigador Titular B, Universidad Nacional Autónoma de México: Coyoacan, Distrito Federal, México

City I Ciudad:

México DF $[\mathrm{mx}]$

e-mail:

ncalleja@unam.mx

Citar como:

Gómez Pérez, O. I., \& Calleja Bello, N. (2017). Regulación emocional: Escalas de medición en español [revisión psicométrica]. Revista Iberoamericana de Psicología issn-l:2027-1786, 10 (2), 183-92.

Obtenido de: https://revistas.iberoamericana.edu.co/index.php/ripsicologia/article/view/1214 


\title{
Regulación emocional Escalas de medición en español [revisión psicométrica]
}

\author{
Emotional regulation: measurement scales in Spanish [Psychometric review] \\ Olimpia Isaura Gómez Pérez \\ Nazira Calleja Bello
}

\section{Introducción}

La regulación emocional, definida como el proceso a través del cual los individuos modulan sus emociones para alcanzar metas, adaptarse al contexto y promover tanto su propio bienestar como el de su grupo social (Gross \& John, 2003), es un fenómeno psicológico sumamente complejo. En sus intentos por regular su experiencia emocional, las personas utilizan diversas estrategias, como modificar la situación elicitadora de la emoción, el contexto, la apreciación, su experiencia subjetiva o su expresión (Gross \& Thompson, 2007). El estudio sistemático de la regulación emocional data de las últimas décadas del siglo XX (Gross J., 1998), principalmente en Estados Unidos. Desde entonces, los autores que se han ocupado de estudiarla han planteado definiciones variadas (para una revisión detallada sobre las definiciones ver (Gómez \& Calleja, 2016) y, en consecuencia, han utilizado distintas formas de evaluar el constructo (Adrian, Zeman, \& Veits, 2011).

Las primeras aproximaciones a la medición de la regulación emocional se centraron en los infantes y los preescolares, mediante el reporte de los padres y los maestros, así como con escalas observacionales de interacción entre diadas. A partir del año 2000 aumentó el número de estudios con niños escolares, en los que se empleaban heteroreportes y escalas observacionales, así como escalas de autoreporte para adolescentes.

Un breve análisis de las investigaciones que evalúan la regulación emocional con niños hispanohablantes ha mostrado que existen sólo algunos estudios con esta población. Esquivel, García, Montero y Valencia (2013) desarrollaron una escala observacional para diadas de madres y sus hijos pequeños; Andrés, Castañeira y Richaud (2014) tradujeron al español la escala Cognitive Emotion Regulation Questionnaire-k, CERQ-k y la aplicaron a niños y niñas de nueve a 12 años; Flórez y Rendón (2010) diseñaron una entrevista estructurada desarrollada a partir de las perspectivas teóricas de Cole, Martin y Dennis (2004), y otros autores, con la que evaluaron a niños de nueve años. Kinkead, Garrido y Uribe (2011) revisaron escalas en inglés de adolescentes y adultos y ubicaron una serie de cuestionarios y pautas de entrevistas que consideran válidas para medir la regulación emocional con dichas poblaciones, entre los que se ubica el Emotion Regulation Questionnaire, ERQ de Gross y John (2003). En los países hispanohablantes, la regulación emocional se ha evaluado en la adolescencia y en la adultez principalmente mediante el autoreporte (Gómez O. , 2013; Sánchez Aragón \& Díaz Loving, 2009).

El desarrollo teórico y empírico de la regulación emocional (así como de cualquier otro constructo) requiere garantizar la calidad de los datos recabados; para ello, es necesario efectuar las mediciones con instrumentos válidos y confiables, que hayan sido sometidos a rigurosos procedimientos psicométricos. En particular, en los países de habla hispana, existe una gran necesidad de conocer los instrumentos que se han utilizado en las investigaciones sobre regulación emocional e identificar su calidad psicométrica.

Los objetivos del presente trabajo fueron:

efectuar una búsqueda metodológica sistemática, actual (del año 2000 al 2015) y exhaustiva a fin de identificar los estudios en los que se reporta la construcción de instrumentos de autorreporte en español para medir la regulación emocional o su adaptación a partir de escalas elaboradas originalmente en idioma inglés

describir las escalas localizadas y, en su caso, sus correspondientes originales en inglés, en términos del propósito del instrumento y la manera en que definen la regulación emocional, la población a la que se dirigen, las dimensiones que evalúan y el número de reactivos determinar la calidad psicométrica de las escalas en términos de los índices de validez y confiabilidad que se reportan identificar los instrumentos que poseen mayor rigor psicométrico para evaluar la regulación emocional en lengua hispana. 


\section{Regulación emocional}

Escalas de medición en español [revisión psicométrica]

El estudio se realizó en tres fases sucesivas: en la primera ubicaron las escalas en español utilizadas en investigaciones publicadas sobre regulación emocional; en la segunda se analizaron los instrumentos seleccionados, y en la tercera se evaluó la calidad psicométrica de los mismos.

\section{Método}

\section{Estrategia de búsqueda}

Para identificar los reportes de elaboración o adaptación de escalas de regulación emocional, se realizaron búsquedas en las siguientes bases de datos electrónicas: Scielo, Redalyc y Tesiunam (base de datos de tesis de la Universidad Nacional Autónoma de México), así como Internet a través de los buscadores Google y Google Scholar.

Las palabras clave utilizadas en la búsqueda fueron: escala de regulación emocional, adaptación de la escala de regulación emocional, así como regulación emocional, escala, cuestionario y medición.

\section{Criterios de inclusión}

Se seleccionaron artículos y tesis, en inglés y en español, publicados del año 2000 al 2015 en revistas indexadas y revisadas por pares, donde se reportará al menos un instrumento de autoreporte para medir regulación emocional, con sus reactivos, dirigidos a niños, jóvenes o adultos.

Tabla 1 Escalas de regulación emocional originales en español (7)

\begin{tabular}{|c|c|c|c|c|}
\hline Código & Escala & MS & $\mathbf{P}$ & Autor(es) \\
\hline CREI & $\begin{array}{l}\text { Cuestionario de Regulación Emocional } \\
\text { Interpersonal de Ira y Tristeza }\end{array}$ & $\begin{array}{c}115 \\
\text { ju }\end{array}$ & es & $\begin{array}{l}\text { (Company, Oberst, \& } \\
\text { Sánchez, 2012) }\end{array}$ \\
\hline $\begin{array}{l}\text { ECPRE- } \\
\text { Amor }\end{array}$ & $\begin{array}{l}\text { Estimación Cognoscitiva de los } \\
\text { Procesos de Regulación Emocional } \\
\text { para la Emoción de Amor }\end{array}$ & $\begin{array}{c}201 \\
\text { ju }\end{array}$ & $m x$ & $\begin{array}{l}\text { (Carrasco Chaves \& } \\
\text { Sánchez Aragón, 2009) }\end{array}$ \\
\hline $\begin{array}{l}\text { ECPRE- } \\
\text { Enojo }\end{array}$ & $\begin{array}{l}\text { Estimación Cognoscitiva de los } \\
\text { Procesos de Regulación Emocional } \\
\text { para la Emoción de Enojo }\end{array}$ & $\begin{array}{l}196 \\
\text { ju }\end{array}$ & $\mathrm{mx}$ & $\begin{array}{l}\text { (Sánchez Aragón \& Díaz } \\
\text { Loving, Identificación } \\
\text { de las estrategias de } \\
\text { regulación emocional para } \\
\text { la frustración y el enojo } \\
\text { mediante una medida } \\
\text { psicométrica, 2009) }\end{array}$ \\
\hline ECPRE- & $\begin{array}{l}\text { Estimación Cognoscitiva de los } \\
\text { Procesos de Regulación Emocional } \\
\text { para la Emoción de Frustración }\end{array}$ & $\begin{array}{l}200 \\
\text { ju }\end{array}$ & $\mathrm{mx}$ & $\begin{array}{l}\text { (Sánchez Aragón \& Díaz } \\
\text { Loving, Identificación } \\
\text { de las estrategias de } \\
\text { regulación emocional para } \\
\text { la frustración y el enojo } \\
\text { mediante una medida } \\
\text { psicométrica, 2009) }\end{array}$ \\
\hline $\begin{array}{l}\text { ECPRE- } \\
\text { Miedo }\end{array}$ & $\begin{array}{l}\text { Estimación cognoscitiva de los } \\
\text { Procesos de Regulación Emocional } \\
\text { para la Emoción de Miedo }\end{array}$ & $\begin{array}{l}184 \\
\text { ju }\end{array}$ & $\mathrm{mx}$ & (Martínez-Pérez, 2009) \\
\hline $\begin{array}{l}\text { ECPRE- } \\
\text { Tris }\end{array}$ & $\begin{array}{l}\text { Estimación Cognoscitiva de los } \\
\text { Procesos de Regulación Emocional } \\
\text { para la Emoción de Tristeza }\end{array}$ & $\begin{array}{l}763 \\
\text { ad }\end{array}$ & $m x$ & $\begin{array}{l}\text { (Sánchez Aragón, Jiménez } \\
\text { Rodríguez, \& Perales } \\
\text { González, 2014) }\end{array}$ \\
\hline EREn & $\begin{array}{l}\text { Escala de Regulación Emocional para } \\
\text { Niños. }\end{array}$ & $\begin{array}{c}190 \\
\text { nñ }\end{array}$ & $m x$ & (Ortiz, 2014) \\
\hline
\end{tabular}

Nota: MS: Muestra; P: País | en muestras ju: jóvenes Universitarios; ad: adultos; nñ: niños | en país (ISO 31166²) es: España; mx: México | Fuente: elaboración

\section{Procedimiento}

Se seleccionaron los artículos de acuerdo con la estrategia de búsqueda y los criterios de inclusión, los cuales fueron verificados por un segundo investigador.
Tabla 2 Escalas de regulación emocional adaptadas al español (17) y las originales en inglés (10)

\begin{tabular}{|c|c|c|c|c|}
\hline Código & Escala & MS & $\mathbf{P}$ & Autor(es) \\
\hline CERQ & $\begin{array}{c}\text { Cognitive Emotion Regulation } \\
\text { Questionnaire }\end{array}$ & $\begin{array}{l}611 \\
\text { ad }\end{array}$ & us & (Garnefski \& Kraaij, 2007) \\
\hline $\begin{array}{l}\text { CERQ- } \\
\text { Esp }\end{array}$ & $\begin{array}{l}\text { Cuestionario de Regulación Emocional } \\
\text { Cognitiva en Español-Adultos }\end{array}$ & $\begin{array}{c}615 \\
j u\end{array}$ & es & $\begin{array}{l}\text { (Domínguez Sánchez, Lasa } \\
\text { Aristu, Amor, \& Holgado } \\
\text { Tello, 2011) }\end{array}$ \\
\hline $\begin{array}{l}\text { CERQ- } \\
\text { Arg }\end{array}$ & $\begin{array}{l}\text { Cuestionario de Regulación Emocional } \\
\text { Cognitiva en Español }\end{array}$ & $\begin{array}{c}359 \\
\text { ju }\end{array}$ & ar & $\begin{array}{l}\text { (Medrano, Moretti, Ortiz, \& } \\
\text { Pereno, 2015) }\end{array}$ \\
\hline $\begin{array}{l}\text { CERQ- } \\
\text { Short }\end{array}$ & $\begin{array}{c}\text { Cognitive Emotion Regulation } \\
\text { Questionnaire } \\
\text { 18-item version }\end{array}$ & $\begin{array}{l}611 \\
\text { ad }\end{array}$ & us & (Garnefski \& Kraaij, 2006) \\
\hline $\begin{array}{l}\text { CERQ- } \\
\text { ShortE }\end{array}$ & $\begin{array}{c}\text { Cuestionario de Regulación Emocional } \\
\text { Cognitiva Versión Corta en Español- } \\
\text { Adolescentes }\end{array}$ & $\begin{array}{c}248 \\
\text { ja }\end{array}$ & es & (Rey \& Extremera, 2011) \\
\hline $\begin{array}{l}\text { CSMS } \\
\text { CAMS } \\
\text { CWMS }\end{array}$ & $\begin{array}{l}\text { Children`s Emotions Management } \\
\text { Scales (Sadness, Anger, Worry) }\end{array}$ & $\begin{array}{c}441 \\
\text { nñ }\end{array}$ & us & $\begin{array}{l}\text { (Zeman, Shipman, \& Penza } \\
\text { Clyve, 2001). } \\
\text { (Zeman, Cassano, Suveg, \& } \\
\text { Shipman, 2010) }\end{array}$ \\
\hline
\end{tabular}

CSMS-

Méx

CAMS-

Méx

CWMS-

Méx

DERS

Escalas de Manejo Emocional de la Tristeza, el Enojo y la Preocupación para Niños

190
$\mathrm{nn}$ $\mathrm{mx} \quad$ (Mendoza, 2010) ing

DERS

Difficulties in Emotion Regulation Scale

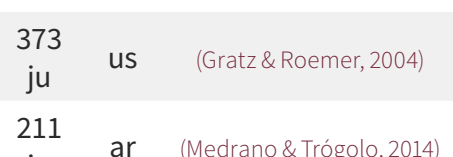

Arg

DERSChile

DERS 2008

DERS

DERS-

DERS-

EROS

EROS Emotion Regulation of Others and Self

EROS- Regulación Emocional de los Otros y Esp

$$
\text { Emotion Regulation Questionnaire }
$$

$$
\text { del Yo }
$$

(Guzmán González,

cl Trabucco, Urzúa, Garrido, \& Leiva, 2014)

1116 ad

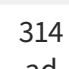

ad

es

455
ae

ae

551

ad

112
ju

es

Hervás \& Jódar, 2008

(Gómez, Penelo, \& De la Osa, 2014)

mx (Marín, Robles, González Forteza, \&Andrade, 2012) (Niven, Totterdell, Stride, \& Holman, 2011)

(Da Costa, Páez, Oriol, \& Unzueta, 2014)

(Gross \& John, Individual differences in two emotion 1628 us regulation processes: ju US implications for affect, relationships and wellbeing, 2003)

ERQ-

2006

ERQ-

Esp

2013

ERQ-

Perú

IEMSS

IEMSS-

Esp

MARS

MARS-

MyE

Cuestionario de Regulación Emocional

en Español

(Cabello, Fernández

Cuestionario de Regulación Emocional en Español

Cuestionario de Autorregulación Emocional

Interpersonal Emotion Management Strategies Scale

Cuestionario de Interregulación Emocional

Measure of Affect Regulation Styles

Medida de Estilos de Regulación Afectiva en Español, ampliada en Ira y Tristeza

$\begin{array}{ccc}\begin{array}{c}166 \\ \text { ju }\end{array} & \text { es } & \begin{array}{c}\text { (Cabello, Fernández } \\ \text { Berrocal, Ruiz-Aranda, \& } \\ \text { Extremera, 2006) }\end{array} \\ \begin{array}{c}\text { 866 } \\ \text { ad }\end{array} & \text { es } & \begin{array}{c}\text { (Cabello, Fernández } \\ \text { Berrocal, \& Gross, 2013) }\end{array} \\ \begin{array}{c}\text { 320 } \\ \text { ju }\end{array} & \text { pe } & \text { (Gargurevich \& Matos, 2010) } \\ 290 & \text { us } & \text { (Little, Kluemper, Nelson, \& } \\ \text { ju } & \text { Gooty, 2011) } \\ 112 & \text { es } & \text { (Da Costa, Páez, Oriol, \& } \\ \text { ad } & \text { Unzueta, 2014) } \\ \text { NR } & \text { us } & \text { (Larsen \& Prizmic, 2004) } \\ 355 & \text { mx } & \text { (Páez, Martínez, Sevillano, } \\ \text { ju } & \text { es } & \text { Mendiburo, \& Campos, }\end{array}$

Nota: MS: Muestra; P: País | en muestras ad: adultos; ae: adolescentes estudiantes; es: estudiantes; ja: adolescentes; ju: jóvenes Universitarios; nñ: niños; NR: No reportado | en país (ISO 31166²) ar: Argentina; cl: Chile; es: España; mx: México; pe: Perú; us: United States (Estados Unidos) | Fuente: elaboración propia 


\section{Resultados}

Las escalas que cumplieron los criterios de inclusión se muestran en orden alfabético en las Tablas 1 y 2. De 7 escalas originales en español, 6 fueron desarrolladas en México (Tabla 1), mientras que las escalas originales en inglés se desarrollaron en Estados Unidos, con sus adaptaciones hechas principalmente en España, así como en México, Perú, Chile y Argentina (Tabla 2).

Enseguida, se ubicó el propósito para el cual fueron construidas y la definición de regulación emocional utilizada. A continuación, se efectuó el análisis psicométrico, utilizando un esquema basado parcialmente en los criterios de calidad planteados por Windle, Bennett y Noyes (2011), expuesto en la Tabla 3. Desde estos criterios, se evaluó la calidad psicométrica de las 34 escalas de regulación emocional seleccionadas.

Tabla 3 Criterios de evaluación de la calidad psicométrica de las escalas

\begin{tabular}{|c|c|c|c|c|}
\hline \multirow{2}{*}{ Propiedad } & \multirow{2}{*}{ Definición } & \multicolumn{3}{|c|}{ Criterios de Calidad según puntaje } \\
\hline & & 0 & 1 & 2 \\
\hline Confiabilidad & $\begin{array}{c}\text { Grado de consistencia en } \\
\text { las respuestas. }\end{array}$ & nd & $\nabla<0.75$ & $\otimes \geq 0.75$ \\
\hline $\begin{array}{c}\text { Otros } \\
\text { índices de } \\
\text { confiabilidad }\end{array}$ & $\begin{array}{l}\text { Pruebas paralelas, } \\
\text { test-retest, división por } \\
\text { mitades }\end{array}$ & nd & $r<0.75$ & $r \geq 0.75$ \\
\hline \multirow{2}{*}{ 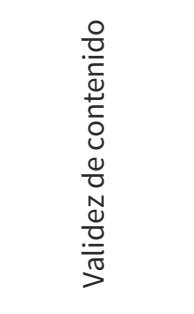 } & \multirow{2}{*}{$\begin{array}{l}\text { Grado en el que la escala } \\
\text { representa todas las } \\
\text { facetas del constructo }\end{array}$} & \multirow[b]{2}{*}{ nd } & \multirow{2}{*}{$\begin{array}{l}\text { Traducción } \\
\text { Retraducción }\end{array}$} & $\begin{array}{l}\text { E. Original: } \\
\text { Jueceo Expertos } \\
\text { Pilotaje }\end{array}$ \\
\hline & & & & $\begin{array}{l}\text { Adaptaciones: } \\
\text { Traducciones } \\
\text { por Expertos } \\
\text { Pilotaje }\end{array}$ \\
\hline 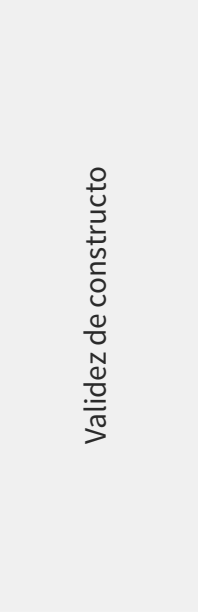 & $\begin{array}{l}\text { Grado en el que } \\
\text { la estructura del } \\
\text { instrumento refleja la } \\
\text { estructura teórica del } \\
\text { constructo }\end{array}$ & nd & $\begin{array}{l}\text { Análisis factorial } \\
\text { exploratorio y/o } \\
\text { confirmatorio }\end{array}$ & $\begin{array}{c}\text { Tamaño } \\
\text { adecuado de } \\
\text { la muestra (7 } \\
\text { personas por } \\
\text { reactivo y } \mathrm{n}> \\
\text { 100), análisis } \\
\text { factorial } \\
\text { exploratorio } \\
\text { con cargas } \\
\text { factoriales }> \\
\text { 0.40 y varianza } \\
\text { explicada > 45\%, } \\
\text { análisis factorial } \\
\text { confirmatorio: } \\
\text { CFI >.90 y RMNSE } \\
<0.05\end{array}$ \\
\hline 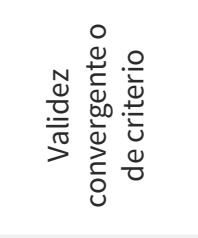 & $\begin{array}{l}\text { Grado en el que los } \\
\text { puntajes del instrumento } \\
\text { se relacionan con otros } \\
\text { instrumentos de una } \\
\text { manera consistente con } \\
\text { la teoría. }\end{array}$ & nd & r significativas & $\begin{array}{c}\text { rentre } \\
\text { constructos } \\
\geq 0.70\end{array}$ \\
\hline $\begin{array}{c}\text { Efectos de } \\
\text { piso y techo }\end{array}$ & $\begin{array}{l}\text { Número de } \\
\text { respondientes que } \\
\text { lograron el puntaje más } \\
\text { alto o más bajo posible. }\end{array}$ & nd & $>15 \%$ & $\leq 15 \%$ \\
\hline 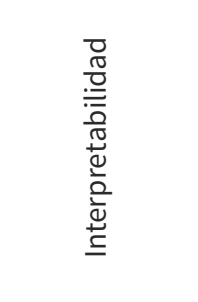 & $\begin{array}{c}\text { Grado en el cual } \\
\text { es posible asignar } \\
\text { significado cualitativo } \\
\text { a los puntajes } \\
\text { cuantitativos. }\end{array}$ & nd & $\begin{array}{l}\text { Presentación } \\
\text { de media y } \\
\text { desviación } \\
\text { estándar de la } \\
\text { muestra }\end{array}$ & $\begin{array}{l}\text { Presentación } \\
\text { de media y } \\
\text { desviación } \\
\text { estándar de } \\
\text { subgrupos } \\
\text { significativos } \\
\text { (género, edad, } \\
\text { grado escolar) }\end{array}$ \\
\hline
\end{tabular}

Fuente: Autores; Ensamble: RIP
Se observó que los propósitos incluyen, por un lado, la medición de estrategias generalizadas como la supresión, la reapreciación o la búsqueda de apoyo social y, por otro, estrategias más específicas, como las estrictamente cognitivas, las que surgen en interacciones sociales, o las destinadas a regular los afectos negativos. Todas las definiciones consideran que la regulación emocional es un proceso intencional para modificar la emoción experimentada, pero varían en las características del esfuerzo: cognitivo vs conductual, extrínseco vs intrínseco, metas personales vs metas sociales.

En la Tabla 4 se muestran las dimensiones que integran a cada instrumento, el número de reactivos para cada dimensión, sus índices de confiabilidad y el número total de reactivos. Posteriormente, en la tabla 5, se presenta una síntesis de las subescalas o dimensiones de las escalas revisadas junto con su porcentaje, organizadas en:

\section{estrategias cognitivas, por ejemplo, reapreciar, concentrarse o planificar \\ estrategias mixtas (cognitivo/conductuales), como distraerse, afrontar o inhibir \\ estrategias conductuales, como suprimir y expresar.}

Lo anterior con el propósito de proponer un agrupamiento general que permita abarcar la gran diversidad y heterogeneidad de estrategias evaluadas por los investigadores.

Tabla 4 Tamaño y tipo de muestra en la que se3 aplicó el instrumento: dimensiones que lo integran, número de reactivos de cada dimensión [NRD] ( índices de confiabilidad de cada dimensión) y número total de reactivos [TRE]

\begin{tabular}{|c|c|c|c|c|}
\hline Código & Dimensiones & NRD & 圆 & TRE \\
\hline \multirow{3}{*}{ CAMS } & Regulación de la ira por afrontamiento & 4 & .73 & \multirow{3}{*}{11} \\
\hline & Inhibición de la ira & 4 & .69 & \\
\hline & Expresión desregulada de la ira & 3 & .68 & \\
\hline \multirow{3}{*}{ CAMS-Méx } & Afrontamiento & 4 & .60 & \multirow{3}{*}{11} \\
\hline & Inhibición & 4 & .57 & \\
\hline & Disregulación & 3 & .51 & \\
\hline \multirow{9}{*}{ CERQ } & Autoculpa & 4 & .75 & \multirow{9}{*}{36} \\
\hline & Aceptación & 4 & .76 & \\
\hline & Rumiación & 4 & .83 & \\
\hline & Reenfocamiento positivo & 4 & .85 & \\
\hline & Reenfocarse en planear & 4 & .86 & \\
\hline & Reapreciación positiva & 4 & .85 & \\
\hline & Poner en perspectiva & 4 & .82 & \\
\hline & Catastrofización & 4 & .79 & \\
\hline & Culpar a otros & 4 & .82 & \\
\hline \multirow{9}{*}{ CERQ-Arg } & Autoculpa & 4 & .69 & \multirow{9}{*}{36} \\
\hline & Aceptación & 4 & .59 & \\
\hline & Rumiación & 4 & .70 & \\
\hline & Reenfocamiento positivo & 4 & .83 & \\
\hline & Reenfocarse en planear & 4 & .66 & \\
\hline & Reapreciación positiva & 4 & .82 & \\
\hline & Poner en perspectiva & 4 & .70 & \\
\hline & Catastrofización & 4 & .77 & \\
\hline & Culpar a otros & 4 & .68 & \\
\hline \multirow{9}{*}{ CERQ-Esp } & Autoculpa & 4 & .60 & \multirow{9}{*}{36} \\
\hline & Aceptación & 4 & .63 & \\
\hline & Rumiación & 4 & .73 & \\
\hline & Reenfocamiento positivo & 4 & .89 & \\
\hline & Reenfocarse en planear & 4 & .79 & \\
\hline & Reapreciación positiva & 4 & .86 & \\
\hline & Poner en perspectiva & 4 & .82 & \\
\hline & Catastrofización & 4 & .71 & \\
\hline & Culpar a otros & 4 & .78 & \\
\hline
\end{tabular}


Regulación emocional

Escalas de medición en español [revisión psicométrica]

\begin{tabular}{|c|c|c|c|c|c|c|c|c|c|}
\hline Código & Dimensiones & NRD & 囚 & TRE & Código & Dimensiones & NRD & 凶 & TRE \\
\hline \multirow{9}{*}{ CERQ-Short } & Autoculpa & 2 & .68 & \multirow{9}{*}{18} & \multirow{5}{*}{$\begin{array}{l}\text { DERS-Esp } \\
2008\end{array}$} & Descontrol & 9 & .91 & \multirow{5}{*}{28} \\
\hline & Aceptación & 2 & .73 & & & Rechazo & 7 & .90 & \\
\hline & Rumiación & 2 & .79 & & & Interferencia & 4 & .87 & \\
\hline & Reenfocamiento positivo & 2 & \multirow{2}{*}{.79} & & & Desatención & 4 & .73 & \\
\hline & Reenfocarse en planear & 2 & & & & Confusión & 4 & .78 & \\
\hline & Reapreciación positiva & 2 & .81 & & \multirow{6}{*}{$\begin{array}{l}\text { DERS-Esp } \\
2014\end{array}$} & Conciencia & 6 & .62 & \multirow{6}{*}{36} \\
\hline & Poner en perspectiva & 2 & .79 & & & Impulso & 6 & .81 & \\
\hline & Catastrofización & 2 & .81 & & & No aceptación & 7 & .84 & \\
\hline & Culpar a otros & 2 & .77 & & & Metas & 5 & .80 & \\
\hline \multirow{9}{*}{$\begin{array}{l}\text { CERQ-Short- } \\
\quad \text { Esp }\end{array}$} & Autoculpa & 2 & .68 & \multirow{9}{*}{18} & & Claridad & 5 & .71 & \\
\hline & Aceptación & 2 & .65 & & & Estrategias & 7 & .77 & \\
\hline & Rumiación & 2 & .64 & & \multirow{4}{*}{ DERS-Méx } & No aceptación & 9 & .85 & \multirow{4}{*}{24} \\
\hline & Reenfocamiento positivo & 2 & .64 & & & Metas & 6 & .79 & \\
\hline & Reentocarse en planear & 2 & .65 & & & Conciencia & 5 & .71 & \\
\hline & Reapreciacion positiva & 2 & .52 & & & Claridad & 4 & .68 & \\
\hline & Poner en perspectiva & 2 & .62 & & & Selección de la situación & 4 & .60 & \\
\hline & $\begin{array}{l}\text { Catastrofización } \\
\text { Culpar a otros }\end{array}$ & 2 & .64 & & & Modificación de la situación & 7 & .76 & \\
\hline & $\begin{array}{l}\text { Culpar a otros } \\
\text { Estrategias dirigidas a cambiarla }\end{array}$ & 2 & .32 & & & Distracción & 5 & .74 & \\
\hline \multirow{6}{*}{ CREI } & $\begin{array}{l}\text { Estrategias dirıgıdas a cambıar la } \\
\text { situación y los lazos sociales }\end{array}$ & 11 & nd & & & Concentración & 9 & .56 & \\
\hline & Estrategias dirigidas a cambiar & & & & & Cambio cognoscitivo & 6 & .55 & \\
\hline & $\begin{array}{l}\text { la atención e interpretación de la } \\
\text { situación }\end{array}$ & 11 & nd & 44 & ECPRE-Amor & $\begin{array}{l}\text { Modulación de la respuesta } \\
\text { incertidumbre }\end{array}$ & & & 150 \\
\hline & Estrategias dirigidas a cambiar la & 11 & nd & & & nerviosismo & & & \\
\hline & respuesta emocional & & & & & racionalización & 119 & $(.61, .91)$ & \\
\hline & $\begin{array}{l}\text { Estrategias dirıgidas a cambiar la } \\
\text { expresión emocional }\end{array}$ & 11 & nd & & & expresión & & & \\
\hline & Regulación de la tristeza por & 5 & .62 & & & optimización y producción & & & \\
\hline CSMS & afrontamiento & & & 12 & & Selección de la situación & 2 & .64 & \\
\hline & Inhibición de la tristeza & 4 & .67 & 12 & & Modificación de la situación & 5 & .60 & \\
\hline & Expresión desregulada de la tristeza & 3 & .60 & & & Distracción & 4 & .66 & \\
\hline & Afrontamiento & 5 & .42 & & $\begin{array}{l}\text { ECPRE- } \\
\text { Enojo }\end{array}$ & Concentración & 4 & .62 & 64 \\
\hline CSMS-Méx & Inhibición & 4 & .60 & 12 & & Cambio cognoscitivo & 6 & $(.68 \& \& .60)$ & \\
\hline & Disregulación & 3 & .45 & & & Modulación de la respuesta & 49 & $(.78, .84$ & \\
\hline & $\begin{array}{l}\text { Regulación de la preocupación por } \\
\text { afrontamiento }\end{array}$ & 3 & .69 & & & Modificación de la situación & 3 & $\begin{array}{l}\& \& .89) \\
60\end{array}$ & \\
\hline CWMS & Inhibición de la preocupación & 4 & .74 & 10 & ECPRE- & Distracción & 4 & .63 & 49 \\
\hline & $\begin{array}{l}\text { Expresión desregulada de la } \\
\text { preocupación }\end{array}$ & 3 & .72 & & & Modulación de la respuesta & 42 & $(.60, .84)$ & \\
\hline & Afrontamiento & 3 & .28 & & & Selección de la situación & 4 & .66 & \\
\hline CWMS-Méx & Inhibición & 4 & 58 & 10 & & Modificación de la situación & 4 & .66 & \\
\hline & Disregulación & 3 & .30 & & Miedo & Despliegue de la atención & 12 & $(.60 \& \& .66)$ & 47 \\
\hline & No aceptación & 6 & & & & Cambio cognoscitivo & 3 & .60 & \\
\hline & Metas & 5 & & & & Modificación de la respuesta & 24 & $(.67, .78)$ & \\
\hline DERS & Conciencia & 6 & $(\bigotimes>.80)$ & 36 & & Selección de la situación & 5 & .67 & \\
\hline & Estrategias & 8 & & & & Desplazamiento de atención & 3 & .70 & \\
\hline & Claridad & 5 & & & ECPRE- & Distracción & 7 & .78 & 143 \\
\hline & Falta de aceptación emocional & 6 & .84 & & & Concentración & 6 & .60 & \\
\hline & $\begin{array}{l}\text { Interferencia en conducta dirigida a } \\
\text { metas }\end{array}$ & 5 & .82 & & & $\begin{array}{l}\text { Cambio cognoscitivo } \\
\text { Modulación de la respuesta }\end{array}$ & $\begin{array}{c}3 \\
59\end{array}$ & $\begin{array}{c}.67 \\
(.61, .87)\end{array}$ & \\
\hline DERS-Arg & $\begin{array}{l}\text { Dificultades en el control de los } \\
\text { impulsos }\end{array}$ & 6 & .87 & 28 & EREn & $\begin{array}{l}\text { Estrategias que facilitan la regulación } \\
\text { emocional }\end{array}$ & 22 & .86 & 33 \\
\hline & Falta de conciencia emocional & 4 & .71 & & & $\begin{array}{l}\text { Estrategias que dificultan la regulación } \\
\text { emocional }\end{array}$ & 11 & .78 & \\
\hline & Falta de claridad emocional & 4 & .71 & & & Mejoramiento intrínseco del afecto & & .82 & \\
\hline & $\begin{array}{l}\text { Acceso limitado a estrategias de } \\
\text { regulación }\end{array}$ & 3 & .54 & & $E P$ & Empeoramiento intrínseco del afecto & & .80 & \\
\hline & Descontrol & 9 & .88 & & ERUS & Mejoramiento extrínseco del afecto & & .82 & 19 \\
\hline & Rechazo & 7 & .89 & & & Empeoramiento extrínseco del afecto & & .74 & \\
\hline DERS-Chile & Interferencia emocional & 4 & .87 & 28 & & Mejora de emociones negativas & 6 & A & \\
\hline & Desatención emocional & 5 & .77 & & EROS-Esp & Empeoramiento de emociones & 3 & S & 9 \\
\hline & Confusión emocional & 3 & .69 & & & negativas & o & 3 & \\
\hline
\end{tabular}


Siete de las escalas se aplicaron a niños, tres a adolescentes, 15 a jóvenes universitarios y nueve a adultos. En algunos casos, la versión adaptada se dirigió a un tipo de población diferente de la original (e. g., CERQ-ShortE). El tamaño de la muestra osciló entre 112 (EROS-ESp) y 1116 (DERS-Chile) participantes (media $\overline{\mathbf{X}}=415.1$; $\mathrm{mdn}=320.0$; de $=322.2$. En promedio, el número de participantes fue más alto en las escalas originales en inglés $(\overline{\boldsymbol{X}}=598.6)$, que en las adaptadas $(\overline{\boldsymbol{X}}=$ 380.1) y que en las originales en español (264.1). El número menor de reactivos en una escala fue de $\mathbf{9}$ (EROS-Esp) y el mayor de $\mathbf{1 5 0}$ (ECPREAmor), con una media de $\mathbf{3 3}$ y mediana de $\mathbf{2 7}$ (de = 32.34).

Las escalas originales en español incluyeron más reactivos $(\bar{x}=$ 75.71), en comparación con las originales en inglés $(\bar{x}=20.80)$ y con las adaptadas $(\bar{x}=22.82)$. El rango del número de dimensiones de las escalas fue de 2 a 23 ( $\bar{x}=5.23$; $m d n=4.0$; de $=3.88$ ), sin diferencias por tipo de escala. Los índices de consistencia interna oscilaron entre $\mathbf{. 2 8}$ y $\mathbf{. 9 1}$ para las dimensiones; sólo para el $\mathbf{2 5 \%}$ de ellas, el Alpha de Cronbach fue a>.80; en algunos casos se indicó sólo el rango de los índices y en otros no se reportaron. El modo de aplicación fue tanto individual como colectivo. El $\mathbf{4 3 . 7 \%}$ de las estrategias medidas tienen que ver con un esfuerzo cognitivo: pensamientos dirigidos a modificar la apreciación de la situación, lo que conlleva un cambio en la emoción misma. Las estrategias de regulación emocional consideradas actualmente por los autores se ven reflejadas en las dimensiones de las escalas analizadas.

Tabla 5 Dimensiones de 33 escalas analizadas*

IEMSS-Esp Reevaluación cognitiva $\quad 5 \quad 26$

agrupadas por tipo de estrategia de regulación emocional, y porcentaje respecto del total de factores o dimensiones $(n=167)$

\begin{tabular}{|c|c|c|c|c|c|}
\hline \multicolumn{2}{|l|}{ Cognitivas } & \multicolumn{2}{|c|}{ Cognitivo/conductuales } & \multicolumn{2}{|l|}{ Conductuales } \\
\hline Dimensiones & $\%$ & Dimensiones & $\%$ & Dimensiones & $\%$ \\
\hline $\begin{array}{l}\text { Reapreciación } \\
\text { o cambio } \\
\text { cognoscitivo }\end{array}$ & 10.8 & $\begin{array}{l}\text { Desatención o } \\
\text { distracción }\end{array}$ & 5.4 & $\begin{array}{l}\text { Modulación de la } \\
\text { respuesta }\end{array}$ & 5.4 \\
\hline $\begin{array}{l}\text { Rumiación o } \\
\text { concentración }\end{array}$ & 6.0 & $\begin{array}{l}\text { No aceptación o } \\
\text { rechazo }\end{array}$ & 3.6 & $\begin{array}{l}\text { Modificación de la } \\
\text { situación }\end{array}$ & 4.8 \\
\hline Aceptación & 3.6 & Afrontamiento & 3.6 & Supresión & 3.6 \\
\hline Reenfocamiento & 3.6 & Inhibición & 3.6 & $\begin{array}{c}\text { Expresión } \\
\text { desregulada } \\
\text { (disregulación) }\end{array}$ & 3.6 \\
\hline Autoculpa & 3.0 & Culpar a otros & 3.0 & $\begin{array}{l}\text { Selección de la } \\
\text { situación }\end{array}$ & 3.0 \\
\hline Planeación & 3.0 & $\begin{array}{l}\text { Despliegue } \\
\text { atencional }\end{array}$ & 3.0 & $\begin{array}{c}\text { Compromiso } \\
\text { comportamental }\end{array}$ & 0.6 \\
\hline Perspectiva & 3.0 & Interferencia & 2.4 & $\begin{array}{l}\text { Ventilar el afecto } \\
\text { negativo }\end{array}$ & 0.6 \\
\hline Catastrofizar & 3.0 & $\begin{array}{l}\text { Orientación a } \\
\text { metas }\end{array}$ & 1.8 & $\begin{array}{l}\text { Mejora intrínseca } \\
\text { del afecto }\end{array}$ & 0.6 \\
\hline $\begin{array}{l}\text { Claridad } \\
\text { emocional }\end{array}$ & 2.4 & Descontrol & 1.8 & $\begin{array}{c}\text { Mejora extrínseca } \\
\text { del afecto }\end{array}$ & 0.6 \\
\hline $\begin{array}{l}\text { Conciencia } \\
\text { emocional }\end{array}$ & 1.8 & Impulso & 0.6 & $\begin{array}{l}\text { Empeoramiento } \\
\text { intrínseco del } \\
\text { afecto }\end{array}$ & 0.6 \\
\hline Estrategias & 1.8 & Distracción pasiva & 0.6 & $\begin{array}{l}\text { Empeoramiento } \\
\text { extrínseco del } \\
\text { afecto }\end{array}$ & 0.6 \\
\hline Confusión & 1.2 & & & $\begin{array}{l}\text { Mejora de } \\
\text { emociones } \\
\text { negativas }\end{array}$ & 0.6 \\
\hline $\begin{array}{l}\text { Compromiso } \\
\text { cognitivo }\end{array}$ & 0.6 & & & $\begin{array}{l}\text { Mejora de } \\
\text { emociones } \\
\text { positivas }\end{array}$ & 0.6 \\
\hline & & & & $\begin{array}{l}\text { Expresión } \\
\text { regulada }\end{array}$ & 0.6 \\
\hline & & & & $\begin{array}{l}\text { Estrategias para } \\
\text { facilitar la RE }\end{array}$ & 0.6 \\
\hline & & & & $\begin{array}{l}\text { Estrategias que } \\
\text { dificultan la RE }\end{array}$ & 0.6 \\
\hline Subtotal & 43.7 & Subtotal & 29.3 & Subtotal & 27.0 \\
\hline
\end{tabular}

$\begin{array}{ll}\text { Supresión emocional } & 5 \\ \text { Expresión regulada } & 6\end{array}$

Distracción activa $\quad 8$

Compromiso cognitivo 5

Compromiso comportamental 4

Rumiación y alejamiento

Esperary revalorar 2

Ventilar el afecto negativo 5

Regulación emocional instrumental 3

Abandono psicológico 4

Apoyo social informativo 2

Apoyo social emocional 2

Apoyo social instrumental 2

Aislamiento social $\quad 2$

Reevaluación positiva 3

Religión 2

Distracción 5

Rumiación 3

Pensamiento desiderativo 1

Regulación fisiológica activa $\quad 2$

Regulación fisiológica pasiva $\quad 4$

Autocontrol o aceptación 3

Autorreconfortarse 3

Humor 1

Cariño 1

Inhibición y supresión 3

Descarga 2

Expresión regulada 


\section{Regulación emocional}

Escalas de medición en español [revisión psicométrica]

Ninguna de las escalas reportó efectos de piso y techo, por lo que este criterio fue eliminado; otros análisis de confiabilidad (además del Alfa de Cronbach) fueron realizados sólo en seis de los 34 casos, por lo que tampoco se consideraron en la evaluación final.

En la Tabla 6 se presentan los resultados del análisis de la calidad psicométrica de las escalas de regulación emocional evaluadas, de mayor a menor puntaje. La mitad de las ellas tuvo una calidad psicométrica intermedia (de 4 a 7 puntos, de un total de 10). Las adaptaciones de la escala $\boldsymbol{D E R S}$ en México, España y Chile, de la $\boldsymbol{E R Q}$ en España y Perú, y la adaptación en Argentina de la escala CERQ, cumplieron cabalmente los criterios establecidos con 8 puntos. La escala $\boldsymbol{E R Q}$ original alcanzó el puntaje más alto de todas las escalas (9).

Tabla 6 Calidad psicométrica de las 34 escalas registradas

\begin{tabular}{|c|c|c|c|c|c|c|}
\hline \multirow[t]{2}{*}{ Escala } & \multicolumn{5}{|c|}{ Criterios de calidad psicométrica } & \multirow[t]{2}{*}{ Total } \\
\hline & Cint & Vcont & vCons & vCONV & Intb & \\
\hline ERQ & 2 & 1 & 2 & 2 & 2 & 9 \\
\hline CERQ-Arg & 1 & 2 & 2 & 1 & 2 & 8 \\
\hline DERS-Chile & 2 & 1 & 2 & 1 & 2 & 8 \\
\hline DERS-E2008 & 2 & 1 & 2 & 1 & 2 & 8 \\
\hline DERS-E2014 & 2 & 2 & 2 & 0 & 2 & 8 \\
\hline DERS-Méx & 2 & 2 & 2 & 2 & 0 & 8 \\
\hline ERQ-Esp2013 & 2 & 1 & 2 & 1 & 2 & 8 \\
\hline ERQ-Perú & 1 & 2 & 2 & 1 & 2 & 8 \\
\hline CAMS & 1 & 1 & 2 & 1 & 2 & 7 \\
\hline CERQ-Esp & 1 & 1 & 2 & 1 & 2 & 7 \\
\hline CSMS & 1 & 1 & 2 & 1 & 2 & 7 \\
\hline CWMS & 1 & 1 & 2 & 1 & 2 & 7 \\
\hline DERS & 2 & 1 & 1 & 1 & 2 & 7 \\
\hline EROS & 1 & 2 & 2 & 1 & 1 & 7 \\
\hline IEMSS & 0 & 2 & 2 & 2 & 1 & 7 \\
\hline CERQ & 2 & 0 & 2 & 1 & 1 & 6 \\
\hline DERS-Arg & 1 & 0 & 2 & 1 & 2 & 6 \\
\hline EREn & 2 & 2 & 1 & 0 & 1 & 6 \\
\hline CERQ-ShortE & 1 & 1 & 0 & 1 & 2 & 5 \\
\hline ERQ-Esp2006 & 0 & 1 & 2 & 1 & 1 & 5 \\
\hline ECPRE Amor & 1 & 1 & 1 & 0 & 1 & 4 \\
\hline ECPRE Enojo & 1 & 1 & 1 & 0 & 1 & 4 \\
\hline ECPRE Frustr & 1 & 1 & 1 & 0 & 1 & 4 \\
\hline ECPRE Miedo & 1 & 1 & 1 & 0 & 1 & 4 \\
\hline ECPRE Tris & 1 & 1 & 1 & 0 & 1 & 4 \\
\hline MARS-MyE & 1 & 1 & 0 & 1 & 1 & 4 \\
\hline CERQ-Short & 1 & 0 & 0 & 1 & 1 & 3 \\
\hline CREI & 0 & 1 & 0 & 1 & 1 & 3 \\
\hline EROS-Esp & 0 & 0 & 1 & 1 & 1 & 3 \\
\hline IEMSS-Esp & 0 & 1 & 0 & 1 & 1 & 3 \\
\hline CAMS-Méx & 1 & 1 & 0 & 0 & 0 & 2 \\
\hline CSMS-Méx & 1 & 1 & 0 & 0 & 0 & 2 \\
\hline CWMS-Méx & 1 & 1 & 0 & 0 & 0 & 2 \\
\hline MARS & 1 & 1 & 0 & 0 & 0 & 2 \\
\hline \multicolumn{4}{|c|}{$\begin{array}{l}\text { Porcentaje de escalas con puntaje } \\
\text { parcial }\end{array}$} & \multicolumn{3}{|c|}{$\begin{array}{l}\text { Porcentaje de escalas por } \\
\text { puntaje total }\end{array}$} \\
\hline 0 & 14.7 & 11.8 & 26.5 & 32.4 & 14.7 & 0-4: 41.2 \\
\hline 1 & 58.8 & 67.6 & 23.5 & 58.8 & 44.1 & 5-7: 35.3 \\
\hline 2 & 26.5 & 20.6 & 50.0 & 8.8 & 41.2 & 8-10: 23.5 \\
\hline Total & 100.0 & 100.0 & 100.0 & 100.0 & 100.0 & 100.0 \\
\hline
\end{tabular}

Nota: Cint: Consistencia Interna; Vcont: Validez del Contenido; vCons: Validez del Constructo; vCONV: Validez Convergente; intb: Interpretabilidad; | 10 originales en inglés, 17 adaptadas al español y 7 originales en español; Fuente: elaboración propia

Por tipo de escala, se encontró que aquellas construidas originalmente en inglés presentaron la media más alta $[\overline{\boldsymbol{X}}=6.2$, rango $(1,9)]$ y una dispersión intermedia $(d e=2.1)$, en tanto que la media de las escalas originales en español fue la más baja (4.1), con la menor dispersión (de =0.9); las escalas adaptadas tuvieron una media de $\mathbf{5 . 6}$ y la desviación estándar más alta (2.5).

El criterio que más cumplieron las escalas analizadas correspondió a la validez de constructo (50\%), y los criterios cubiertos parcialmente o no cubiertos fueron los relativos a la validez convergente y a la de contenido.

\section{Discusión y conclusiones}

El presente estudio tuvo como propósito revisar sistemáticamente las propiedades psicométricas de las escalas de medición de la regulación emocional para utilizarse en población hispanohablante. Se encontró que las escalas analizadas fueron congruentes con las definiciones más representativas de regulación emocional, que sitúan al constructo como un proceso, individual o colectivo, en el que se ponen en práctica estrategias, ya sea de forma consciente o automática, para modificar uno o varios de los componentes del proceso de generación de la emoción con el propósito de cambiar tanto la experiencia como la respuesta emocional, ya sea propia o ajena.

Las estrategias de regulación emocional que evalúan las escalas analizadas son muy diversas, como se observa al analizar los propósitos de éstas. En sus objetivos, los investigadores determinan: a) el componente del proceso de generación de la emoción al que va dirigido la estrategia (por ejemplo, fisiología o apreciación); b) el tipo de estrategia (cognitiva, conductual o mixta); c) el propósito (individual o social), y d) las características de los individuos (e.g., niños, adolescentes, adultos). Esta multiplicidad evidencia las dificultades que entraña medir todas las estrategias de regulación emocional en distintos contextos, individuos y para diversas emociones y sus componentes. Porejemplo, las escalas ECPRE (SánchezAragón\& DíazLoving, 2009), comparten el marco teórico propuesto por Gross y Thompson (2007); sin embargo, una vez realizados los análisis para cada escala, no aparecieron todas las estrategias para todas las emociones, como en el caso de la frustración, lo cual muestra que las estrategias de regulación emocional dependen de la emoción que se está experimentando.

Aunque la regulación emocional se define como un conjunto muy amplio de pensamientos, sentimientos y conductas, la reapreciación o cambio cognoscitivo resultó la estrategia más evaluada. Lo anterior puede deberse a que se considera que las estrategias reapreciativas son más efectivas pues modifican la emoción en su totalidad y no sólo su expresión, como en el caso de las estrategias supresivas (Gross \& John, 2003); por lo que pueden ser más representativas de lo que implica regular adecuadamente las emociones.

Se observaron otras estrategias, como el rechazo y la aceptación, el reenfocamiento, el afrontamiento, la inhibición y la expresión mal regulada o disregulación, cuya presencia en los instrumentos analizados disminuyó paulatinamente hasta las que aparecen en un solo instrumento, como las que miden regulación emocional interpersonal.

Las estrategias de este último tipo (interpresonal): mejora intrínseca del afecto, mejora de emociones positivas y negativas o expresión regulada, se han comenzado a estudiar de manera reciente y su medición se vuelve más compleja porque es necesario considerar las interacciones de las personas, su contexto y sus propósitos sociales 
(Robinson, 2014). Puede ser debido a lo anterior que las estrategias interpersonales de regulación emocional aparecieron muy poco en las escalas consideradas.

Se encontraron muy pocas escalas de autorreporte para niños, tres en inglés para las emociones de frustración, ira y tristeza, y una en español. De acuerdo con (Calkins \& Leerkes, 2011), la regulación emocional es un hito del desarrollo y en la infancia todavía se encuentra en proceso, apoyada por la corregulación de otros; por tanto, la mayoría de los instrumentos de regulación emocional en la infancia son de tipo observacional de diadas o en formato de entrevista a padres y maestros (Adrian, Zeman, \& Veits, 2011). Las escalas de autorreporte deben ir dirigidas a poblaciones de adolescentes y adultos, quienes poseen estrategias de regulación más definitivas y pueden reconocerlas más fácilmente.

Además de analizar las escalas adaptadas y originales en español, se revisaron las escalas originales en inglés correspondientes; con el propósito de comparar sus características. En general, se observó que la calidad psicométrica de las escalas originales es mayor que la de las adaptaciones. Lo anterior puede deberse a diversos motivos, por un lado, las escalas en inglés están mayormente vinculadas con la teoría, ya que la investigación en el campo se ha desarrollado principalmente en países anglosajones, resultando en mejores índices de validez de contenido y de constructo. También se observó que, en su mayoría, las escalas originales en inglés se desarrollaron con un tamaño de muestra mayor, por lo que sus índices de confiabilidad pueden aumentar en comparación con escalas desarrolladas con muestras más pequeñas (Muñiz, 1998).

Por otra parte, el componente cultural en la regulación emocional es muy fuerte. Las culturas anglosajonas son diferentes de las latinoamericanas, ocasionando que un mismo reactivo de regulación emocional sea interpretado de manera distinta por los respondientes. Por ejemplo, - evito demostrar mis sentimientos- puede ser un indicador del uso de una estrategia supresiva de la emoción en individuos latinos, mientras que es una conducta común en individuos de países anglosajones.

Finalmente, se encontró que las escalas adaptadas al español no pasaron, en su mayoría, por un proceso de validación de contenido por jueces expertos (a excepción de la escala DERS), lo que ocasionó una disminución en su puntaje final de calidad psicométrica. A partir de los hallazgos anteriores, se puede recomendar que las escalas adaptadas al español pasen por un juicio de expertos tanto en el constructo como en la cultura de la población a la que se adaptan, aunque se deriven de escalas con índices adecuados de calidad psicométrica.

La diversidad de los instrumentos que existen para medir la regulación emocional dan la posibilidad a los investigadores de seleccionar aquellos que consideren adecuados para sus propósitos; sin embargo, es importante revisar no sólo el o los tipos de estrategias que se pretenden medir, sino también la calidad psicométrica de los instrumentos; evitando utilizar sólo aquellos que se han popularizado, aunque únicamente cumplan con los criterios psicométricos mínimos.

Las cuatro escalas originales en inglés más utilizadas hasta el momento, que oscilaron entre los seis y ocho puntos de calidad psicométrica en el presente análisis, miden la regulación de emociones únicamente negativas (ira, tristeza y preocupación), como las escalas CEMS, CWMS y CAMS, la regulación de tipo cognitiva (CERQ), la regulación disfuncional o desadaptativa (DERS), o sólo las estrategias de supresión y reapreciación (ERQ).

De las tres escalas restantes más utilizadas actualmente en estudios en inglés, la Affective Control Scale y la Regulatory Emotional Self-Efficacy Scale miden a la regulación emocional como un todo, lo cual impide considerar los matices del constructo, como las estrategias que se ponen en práctica según el tipo de emoción, la actividad que se está realizando, las personas que acompañan o el lugar en el que se encuentra el individuo.

Un aspecto más que dificulta la popularización de las escalas es que algunos autores no incluyen todos los reactivos que las integran en los artículos que reportan los estudios de validación. Tal fue el caso de la mayoría de los artículos localizados en la primera fase del presente estudio, ya que de los $\mathbf{1 6 7}$ estudios en los que se utilizaban instrumentos para evaluar la regulación emocional, cerca del $\mathbf{7 5} \%$ de ellos no presentaba la escala completa. Esta carencia obstaculiza la elección de instrumentos adecuados para la realización de los estudios, ya que no es posible determinar si los reactivos son congruentes con cierto marco teórico o si el fraseo de los mismos corresponde a determinada población. Asimismo, el no contar con la totalidad de los reactivos de las escalas dificulta la realización de análisis psicométricos como el presente. El no publicar las escalas completas atenta contra la replicabilidad de los estudios y la divulgación científica.

Por lo anterior, se recomienda, tanto a los investigadores de la regulación emocional como para estudios posteriores de la calidad psicométrica de escalas que miden el constructo, contar con las escalas completas, que pueden ser localizadas directamente con los autores, de tal forma que, aunque se ubiquen menos escalas, puedan someterse todos los reactivos y dimensiones al análisis.

Respecto de los índices psicométricos reportados en las escalas analizadas, se encontró que las adaptadas al español parecen ser menos confiables que las originales en inglés, aunque no en todos los casos. Las adaptaciones del ERQ en México (Cabello, Fernández Berrocal, \& Gross, 2013), España (Cabello, Fernández Berrocal, Ruiz-Aranda, \& Extremera, 2006) y Perú (Gargurevich \& Matos, 2010) conservaron una confiabilidad >.70 para ambas subescalas; mientras que en las adaptaciones de la DERS es posible encontrar subescalas con índices bajos $(\mathbf{. 5 4} \circ \mathbf{. 6 2}$, por ejemplo). Para el caso de la escala $\mathbf{C E R Q}$, en sus adaptaciones para España y Argentina, se redujo la confiabilidad en las mismas subescalas: autoculpa y aceptación. La confiabilidad también se redujo en las adaptaciones de las CAMS, CWMS, CSMS y MARS. El hecho de que las adaptaciones parezcan menos confiables que las escalas originales puede deberse a que:

no todas las traducciones fueron juzgadas por expertos las escalas sufrieron modificaciones en cuanto al número de reactivos el tamaño de la muestra fue reducido.

Es de notar que los puntajes de calidad psicométrica fueron más altos para los instrumentos originales en inglés. Sólo la escala DERS adaptada para adolescentes mexicanos cumplió los criterios de confiabilidad y validez necesarios para ser aplicada en dicha población (ocho puntos de 10). La escala original en español para niños EREn, elaborada como parte de un trabajo de tesis de licenciatura en México, cumple parcialmente con los criterios (seis puntos de 10), con buenos índices de consistencia interna y de validez de contenido, pero no se correlacionó con otras medidas para obtener su validez convergente. Las escalas originales en español más conocidas son las ECPRE para las diferentes emociones; sin embargo, el hecho de que no alcance niveles adecuados de confiabilidad y validez (cuatro puntos de 10), así como su uso en poblaciones diferentes a la de universitarios, limita la calidad de las conclusiones que pueden derivarse de su aplicación. Estos resultados constituyen un llamado a los investigadores hispanohablantes para que desarrollen instrumentos con mayor rigor psicométrico.

De las subescalas que se identificaron, aquellas con índices 


\section{Regulación emocional}

Escalas de medición en español [revisión psicométrica]

de confiabilidad más altos ( $a \geq 75$ ) fueron: reapreciación o cambio cognoscitivo, rumiación o concentración, desatención o distracción, modificación de la situación, no aceptación o rechazo, aceptación, reenfocamiento positivo, autoculpa, reenfocarse en la planeación, poner en perspectiva, catastrofizar, culpar a otros, claridad emocional, orientación a metas, conciencia emocional, estrategias, mejora instrínseca y extrínseca del afecto, empeoramiento intrínseco del afecto, estrategias para facilitar la regulación emocional y estrategias que la dificultan. Estas subescalas son consistentes con el constructo que se pretende medir, por lo tanto, un buen instrumento podría ser el que incorpore como subescalas a las estrategias anteriores para medir la regulación emocional.

Asimismo, también tendrían que considerarse en las limitaciones o direcciones futuras de los estudios los aspectos culturales y sociales, como el lenguaje o el nivel socioeconómico, variables individuales como el grado de estudios, el sexo, e incluso la emoción que se está experimentando; que sean distintos a los considerados en las escalas utilizadas para medir.

La identificación y utilización de escalas existentes para medir los constructos de interés facilita el proceso de investigación y permite la optimización del tiempo y los recursos; sin embargo, es necesario seleccionar las escalas no sólo por su correspondencia con los propósitos de la investigación, sino también por sus características psicométricas a fin de garantizar una adecuada toma de decisiones.

Los análisis que se reportan en este trabajo fueron realizados con el fin último de conocer las propiedades psicométricas de escalas disponibles actualmente para medir la regulación emocional en población hispanohablante. De este esfuerzo pueden desprenderse las siguientes recomendaciones para estudios futuros que tengan el propósito de evaluar la calidad psicométrica de las escalas disponibles que midan un constructo en particular: establecer previamente los elementos que se evaluarán (confiabilidad, validez, interpretabilidad), construir la red nomológica del constructo que señale las principales variables con las que se relaciona, obtener los datos psicométricos con precisión y determinar en qué medida se han cumplido los criterios de calidad psicométrica.

En suma, los resultados del presente estudio conducen a concluir que no se cuenta con escalas originales en español para medir la regulación emocional con niveles altos de calidad psicométrica en las categorías de consistencia interna, validez de contenido, validez de constructo, validez convergente e interpretabilidad, por lo que es fundamental que los investigadores de este constructo desarrollen o adapten escalas con características psicométricas adecuadas y que las reporten a fin de garantizar la validez de sus hallazgos; así como informar la metodología seguida para garantizar la replicabilidad de los estudios y la divulgación científica.

La investigación en el campo de la medición de la regulación emocional aún tiene que responder algunas cuestiones: ¿qué tipo de escalas son mejores para diferentes poblaciones?, ¿cuáles criterios de calidad psicométrica son más pertinentes para la evaluación de las escalas?, ¿cómo se puede mejorar la calidad psicométrica de las escalas desarrolladas en el idioma español?, ¿las estrategias o subescalas incorporadas en los instrumentos de medición deben depender de la cultura? El desarrollo de más y mejores instrumentos para la evaluación confiable y válida de la regulación emocional apoyará el incremento en la evidencia empírica para responder estas y otras preguntas.

\section{Referencias}

Adrian, M., Zeman, J., \& Veits, G. (2011). Methodological implications of the affect revolution: A 35-year review of emotion regulation assessment in children. Journal of Experimental Child Psychology, 110, 171-197. Obtenido de https://www.sciencedirect.com/science/article/pii/S0022096511000907

Andrés, M. L., Castañeira, C. E., \& Richaud, M. C. (2014). Relaciones entre la personalidad y el bienestar emocional en niños. El rol de la regulación emocional. Panamerican Journal of Neuropsychology, 8(2), 217-241. Obtenido de http://www.cnps.cl/index.php/cnps/article/view/169

Cabello, R., Fernández Berrocal, P., \& Gross, J. (2013). A Spanish Adaptation of the Emotion Regulation Questionnaire. European Journal of Psychological Assessment, 29, 234-240. Obtenido de https://econtent.hogrefe.com/doi/ abs/10.1027/1015-5759/a000150

Cabello, R., Fernández Berrocal, P., Ruiz-Aranda, D., \& Extremera, N. (2006). Una aproximación a la integración de diferentes medidas de regulación emocional. Ansiedad y Estrés, 30(1), 14-29. Obtenido de http://emotional. intelligence.uma.es/documentos/1-Cabello2006.pdf

Calkins, S., \& Leerkes, E. (2011). Early attachment processes and the development of emotional self-regulation. Nueva York [us]: Guilford.

Carrasco Chaves, E., \& Sánchez Aragón, R. (2009). Validación de la Estimación Cognoscitiva de los Procesos de Regulación Emocional para la Emoción de Amor. Interpersona, 3(1), 14-29. Obtenido de https://interpersonaabpri. files.wordpress.com/2010/12/paper-2 validacic3b3n-de-la-estimacic3b3n. pdf

Cole, P., Martin, S., \& Dennis, T. (2004). Emotion regulation as a scientific construct: Challenges and directions for child development research. Child development, 75, 317-333. Obtenido de http://urban.hunter.cuny edu/ tdennis/pdf-files/ER scientif_construct_CD.pdf

Company, R., Oberst, U., \& Sánchez, F. (2012). Regulación emocional interpersonal de las emociones de ira y tristeza. Boletín de Psicología, 104, 7-36. Obtenido de https://wwW.uv.es/seoane/boletin/previos/N104-1.pdf

Da Costa, S., Páez, D., Oriol, X., \& Unzueta, C. (2014). Regulación de la afectividad en el ámbito laboral: Validez de las escalas de hetero-regulación EROS y EIM. Journal of Work and Organizational Psychology, 30, 13-22. Obtenido de https://wWw.sciencedirect.com/science/article/pii/S157659621470026X

Domínguez Sánchez, F. J., Lasa Aristu, A., Amor, P. J., \& Holgado Tello, F. P. (2011). Psychometric Properties of the Spanish Version of the Cognitive Emotion Regulation Questionnaire. Assessment, 20, 253-261. Obtenido de https:// www.ncbi.nlm.nih.gov/pubmed/21467092

Esquivel, M., García, B., Montero, M., \& Valencia, A. (2013). Regulación maternal y esfuerzo de control emocional en niños pequeños. International Journal of Psychological Research, 6(1), 30-40. Obtenido de http://www.scielo.org. co/pdf/ijpr/v6n1/v6n1a05.pdf

Flórez, L., \& Rendón, M. I. (2010). Impacto de las reglas de despliegue sobre la regulación emocional en una muestra de escolares. Acta Colombiana de Psicología, 13(2), 79-90. Obtenido de http://editorial.ucatolica.edu.co/ ojsucatolica/revistas ucatolica/index.php/acta-colombiana-psicologia/ article/view/372

Gargurevich, R., \& Matos, L. (2010). Propiedades psicométricas del cuestionario de Autorregulación Emocional Adaptado para el Perú (ERQP). Revista Psicológica, 12(1), 53-67. Obtenido de http://revistas.ucv.edu.pe/index. php/R PSI/article/view/335

Garnefski, N., \& Kraaij, V. (2006). Cognitive emotion regulation questionnairedevelopment of a short 18-item version (CERQ-short). Personality and Individual Differences, 41(6), 1045-1053. Obtenido de https://WwW. sciencedirect.com/science/article/pii/S0191886906001565

Garnefski, N., \& Kraaij, V. (2007). The Cognitive Emotion Regulation Questionnaire. Psychometric Features and Prospective Relationships with Depression and Anxiety in Adults. European Journal of Psychological Assessment, 23, 141-149. Obtenido de https://econtent.hogrefe.com/doi/abs/10.1027/10155759.23.3.141

Gómez, I., Penelo, E., \& De la Osa, N. (2014). Estructura factorial e invariancia de la Escala de Dificultades en la Regulación Emocional (DERS) en adolescentes españoles. Psicothema, 26(3), 401-408. Obtenido de https:// psiquiatria.com/psicologia/estructura-factorial-e-invariancia-de-laescala-de-dificultades-en-la-regulacion-emocional-ders-en-adolescentesespanoles/ 


\section{Regulación emocional}

Escalas de medición en español [revisión psicométrica] 